\title{
Prevalence of maternal alloantibodies in a large teaching hospital and their
}

impact on outcomes of foetuses/neonates

Louise Ainley ${ }^{1}$, Jessica R Jardim ${ }^{1}$, Joachim $\operatorname{Tan}^{2}$, Shohreh Beski ${ }^{1}$, Eva Maria RiosLeal ${ }^{1}$, Shubha Allard ${ }^{1,3}$ Laura Green ${ }^{1,2,3}$

${ }^{1}$ Barts Health NHS Trust

${ }^{2}$ Barts and the London School of Medicine and Dentistry, Queen Mary University of London

${ }^{3}$ NHS Blood and Transplant

Correspondence to:

Dr Laura Green

NHS Blood and Transplant and Barts Health NHS Trust

Charcot Road

Colindale

$N W 95 B G$

Email: laura.green@nhsbt.nhs.uk 


\section{Introduction}

The incidence and severity of haemolytic disease of the newborn (HDN) has reduced significantly since the introduction of prophylactic anti-D in the late 1960s (Mackenzie et al. 1999). UK guidelines recommend that pregnant women should have ABO/D grouping and antibody screening at 10-16 weeks and at 28 weeks of pregnancy (White et al. 2016; NICE guidelines 2015). For those who have anti-D, anti-c and anti-K laboratory monitoring should be continued monthly up to 28 weeks, and fortnightly thereafter (White et al. 2016;). If maternal alloantibody levels rise significantly during pregnancy referral to a foetal medicine unit is recommended. The foetus is monitored for signs of anaemia using Doppler ultrasonography, which measures the peak systolic velocity of the middle cerebral artery (MCA-PSV). At birth, infants born to women who have clinically significant antibodies are examined for signs of HDN and DAT from the cord sample is performed: if this is positive haemoglobin and bilirubin levels are measured.

The aims of this retrospective study were to estimate the incidence of maternal, clinically significant alloantibodies in a large tertiary referral centre, characterise their specificity, describe the clinical outcome of the foetus/neonates and identify areas for change.

\section{Method}

The study met criteria for service evaluation under the Research Ethics Service guidance, so no research and ethical review approval was required. Data from transfusion laboratory information system (Winpath Version 5) was used to identify pregnant women who had clinically significant antibodies between 2003 and 2013, while foetal/neonatal outcome were collected from patients' electronic records and clinical notes for the years 2008-2013.. The ABO/RhD blood grouping and antibody screening of women were checked at booking and 28 weeks as recommended by national guidelines (White et al 2016). ABO grouping and antibody identification were determined using Immucor NEO analyzer, a closed system that utilizes CAPTURE® technology (solid phase) to identify clinically significant antibodies (IBG Immuncor Ltd.4, Riverside Business Centre, Brighton, BN43 6RE). 
Data for Doppler MCA-PSV results were collected from the foetal maternal unit using antenatal clinic record system. This included all MCA-PSV ultrasound results (Vmax) and ultrasound opinions on whether the scan showed evidence of foetal anaemia or hydrops.

\section{Definitions:}

Antibodies were divided into the following groups (Chandrasekar et al. 2001):

Group-1: anti-D, anti-c and anti-K

Group-2: anti-Fy ${ }^{\mathrm{a}}$, anti-Fy ${ }^{\mathrm{b}}$, anti-Jk ${ }^{\mathrm{a}}$, anti-Jk ${ }^{\mathrm{b}}$, anti-C, anti-Ce, anti-e, anti-E, anti-C ${ }^{\mathrm{w}}$

Group-3: anti-M, anti-N, anti-Kpa, anti-S, anti-Le ${ }^{\mathrm{a}}$ and anti-Le ${ }^{\mathrm{b}}$ (non-significant as don't cross placenta)

Neonatal anaemia was defined as:

- $\quad$ at birth, capillary Haemoglobin $(\mathrm{Hb})$ of $<$ or $=$ to $14.0 \times 10^{9} \mathrm{~g} / \mathrm{dl}$

- $\quad$ at less than a week old, $\mathrm{Hb}<$ or $=$ to $15.0 \times 10^{9} \mathrm{~g} / \mathrm{dl}$

- $\quad$ at 2 weeks and under, $\mathrm{Hb}<$ or $=$ to $13.5 \times 10^{9} \mathrm{~g} / \mathrm{dl}$

\section{Results}

From 2003 to 2013 we identified 154 women with clinically significant maternal alloantibodies (out of 21,665 women), giving a prevalence of $0.71 \%$ (95\% CI $0.60-0.82$ ). The most common were anti-E (23\%), anti-D (12\%), anti-K (9\%) and anti-c (8\%).

\section{Foetal/ Neonatal outcomes}

We excluded fifteen women due to terminations, miscarriages, ectopic pregnancies and still births (unrelated to HDN), leaving 139 cases. $92 \underline{(66 \%)}$ had previous pregnancies and $8 \underline{(6 \%)}$ acquired the alloantibodies from blood transfusion ( 4 anti-E, 2 anti-K, 1 anti-D + anti-K, and 1 anti-c). Paternal testing was performedin 54/73 (74\%) cases this was found to be the cause of maternal alloantibodies.

There were no foetal or neonatal deaths attributed to maternal red cell alloimmunisation. Due to significant increase in alloantibody levels 49 (ㅎ5\%) women underwent Doppler ultrasound MCA-PSV, and six (12\%) were diagnosed as having foetal anaemia. There was one intrauterine transfusion at 32 weeks, the rest delivered urgently (two at 34 weeks, two at 36 weeks, one unknown). One neonate was subsequently treated for sepsis post-delivery. 
In total there were 124 babies delivered (15 missing), of these 25 (20\%) were anaemic. Direct antiglobulin test (DAT) was positive in $49 / 109$ (45\%) at delivery with the most frequent alloantibodies being anti-D $(n=16)$, anti-E $(n=16)$, other group- 2 antibodies $\underline{(n=15, D C, J k b}$ $\underline{\mathrm{CD}, \mathrm{Jka}, \mathrm{E} \mathrm{Jkb})}$ anti-c (n=9).

Of the neonates that were found to be anaemic, DAT was positive in $21 / 25(84 \%)$, and the eluate confirmed maternal alloantibodies as the cause for anaemia in these babies. Three $(12 \%)$ had exchange transfusions and nine $(36 \%)$ required top up transfusions. Before discharge 15 more babies became anaemic (12\%). The development of anaemia ranged from day one to 32 ; two of these babies required top-up transfusion.

\section{Discussion}

The prevalence of maternal clinical significant antibodies in our institution was $0.7 \%$, with the $\mathrm{Rh}$ system being the most common antibodies. These results are consistent with other studies (Smith et al. 2015; Pal et al. 2013; Foudoulaki-Paparizos et al. 2013). Of six cases that were diagnosed with foetal anaemia by US Doppler, five were due to $\mathrm{Rh}$ antibodies and one to anti-K. Current UK guidelines recommend that regular monitoring is performed only for anti-D, anti-K and anti-c antibodies (White et al. 2016). Our results, and those of others, suggest that regular laboratory monitoring could be justified for all Rhesus antibodies, and in particular for anti-E antibody (Smith et al. 2015; Fan et al. 2014; Pal et al. 2013).

While the overall incidence of anti-D has reduced significantly with the introduction of immune prophylaxis, it is still the single most common cause of neonatal morbidity. In this study anti-D accounted for $40 \%$ of neonatal anaemia, $55 \%$ of top up transfusions, $33 \%$ of exchange transfusions and the only case that required intrauterine transfusion.

In the UK it is recommended that all women of childbearing age are transfused $\mathrm{Rh} \mathrm{D}$ and $\mathrm{K}$ compatible red cells. In this study only two out of eight cases that we identified to have acquired antibodies from previous transfusion cases were due to anti-K, and anti-K plus anti$\mathrm{D}$, suggesting that this has had a positive impact on outcome. A potential explanation for these two cases may be transfusion abroad. 
Another important finding was the development of neonatal anaemia after birth in $12 \%$ of cases. As current guidelines only recommend a blood test at delivery a significant minority of neonatal anaemia may be missed. It was not possible to comment specifically on the time course of when this developed from our data as monitoring of neonatal haemoglobin was variable and intermittent. Further haemoglobin monitoring during the neonatal period in babies with maternal alloantibodies could be introduced to avoid missing this group.

We identified two outcomes to recommend a change in practice. Firstly, that adverse neonatal outcomes were associated with all Rhesus groups and we suggest that regular laboratory monitoring in pregnancy should be carried out on all these groups, particularly anti-E. Secondly, a significant proportion of neonates developed anaemia after delivery and may be missed if following current guidelines. Further work is needed to evaluate the time course of this and to identify an appropriate monitoring schedule. 


\section{Acknowledgements}

Laura Green and Shoreh Beski designed the study and wrote the manuscript.

Joachim Tan analysed the data.

Louise Ainley analysed the data and wrote the manuscript.

Jessica R Jardim, Eva Maria RiosLeal and Shuba Allard collected the data.

Competing interests: the authors have no competing interests 
(1) Chandrasekar A, Morris KG, Tubman TR, Tharma S, McClelland WM. (2001) The clinical outcome of non-RhD antibody affected pregnancies in Northern Ireland. Ulster Med J, 70(2):89-94.

(2) Fan J, Lee BK, Wikman AT, Johansson S, Reilly M. (2014) Associations of Rhesus and nonRhesus maternal red blood cell alloimmunization with stillbirth and preterm birth. Int $J$ Epidemiol, 43(4):1123-31.

(3) Foudoulaki-Paparizos L, Valsami S, Bournas N, Tsantes A, Grapsas P, Mantzios G, et al. (2013) Alloimmunisation during pregnancy in Greece: need for nationwide HDFN prevention programme. Transfus Med, 23(4):254-9.

(4) White J, Qureshi H, Massey E, et al. (2016) Guideline for blood grouping and antibody testing in pregnancy. Transfus Med, 26(4):246-263.

(5) MacKenzie IZ, Bowell P, Gregory H, Pratt G, Guest C, Entwistle CC. (1999)Routine antenatal Rhesus D immunoglobulin prophylaxis: the results of a prospective 10 year study. Br J Obstet Gynaecol, 106(5):492-7.

(6) Milkins C, Berryman J, Cantwell C, Elliott C, Haggas R, Jones J, et al. (2013) Guidelines for pre-transfusion compatibility procedures in blood transfusion laboratories. British Committee for Standards in Haematology. Transfus Med, 23(1):3-35.

(7) National Institute for Health and ClinicalExcellence (NICE CG62). Antenatal Care, Routine care for the healthy pregnant woman. URL http://www nice org uk/nicemedia/live/11947/40115/40115 pdf 2008;Accessed on 10.02.2015.

(8) Pal M, Williams B. (2015) Prevalence of maternal red cell alloimmunisation: a population study from Queensland, Australia. Pathology, 47(2):151-5.

(9) Smith HM, Shirey RS, Thoman SK, Jackson JB. (2013) Prevalence of clinically significant red blood cell alloantibodies in pregnant women at a large tertiary-care facility.

Immunohematology, 29(4):127-30. 


\begin{tabular}{l|c|c|}
\hline Neonatal Outcome (n= denominator) & Cases & Comments \\
\hline $\begin{array}{l}\text { Ultrasound Doppler MCA PSV raised } \\
(\mathbf{n}=\mathbf{4 9 )}\end{array}$ & $6(12.2 \%)$ & $\begin{array}{c}2 \text { anti-D, } 1 \text { anti-c, } 1 \text { anti-E and anti-c, } \\
1 \text { anti-K, and } 1 \text { anti-D, anti-Jkb and } \\
\text { anti-c. }\end{array}$ \\
\hline Intra uterine transfusion & $1(2 \%)$ & Anti-D \\
\hline Anaemic (n=82) & & $\begin{array}{c}9 \text { anti-D, } 4 \text { anti-D + other, } 3 \text { anti-E, } 1 \\
\text { anti E + other, } 6 \text { group }-2 \text { Ab, } 4 \text { anti- } \\
\text { c, } 2 \text { anti-E, } 1 \text { anti-K and } 1 \text { group-3 }\end{array}$ \\
\hline
\end{tabular}

$\mathrm{Ab}$

\begin{tabular}{lcc}
\hline Raised bilirubin (n=82) & $28(60 \%)$ & \\
\hline DAT Positive (n=109) & $49(45 \%)$ & $42 / 49$ had maternal Ab detected by \\
& & eluate \\
\hline Top up Transfusion (n=124) & $9(6.5 \%)$ & 4 anti-D, 3 anti-D +other, 2 anti-E \\
\hline Exchange Transfusion (n 124) & $3(2 \%)$ & 1 anti-D, 1 anti-E, 1 anti-D/anti-C
\end{tabular}

Footnote: The denominator varies for several reasons, including some for which data were 'not available' 
Table 1: $\quad$ Foetal and Neonatal outcome data 